'Ş Inci Yolal, ${ }^{2} Y$ Karal, ${ }^{2} S$ Karasalihoğlu, ${ }^{3 B}$ Tokuç. 'Department of Pediatrics, ${ }^{2}$ Department of Pediatrics, Division Pediatric Neurology; ${ }^{3}$ Department of Public Health Medical Faculty of Trakya University, Edirne, Turkey

Aim Sleep disturbances are frequently seen in the epileptic patients. This may be because of either own epilepsy, or by chance, or because of the antiepileptics used. We tried to figure out the effects of epilepsy and antiepileptic drugs on the sleep structure.

Methods We questioned 35 epileptic patients, who are followed up by Medical Faculty of Trakya University, Department of Pediatrics, Division Pediatric Neurology, and their 35 healty siblings with Pediatric Sleep Questionnaires which were filled up by patients' families. Patients' structures of sleep were evaluated by asking the following questions regarding behaviours in night sleep and daytime sleep, behaviours in daytime, attention deficit disorder with hyperactivity.

Results We have ascertained that totally questionnaire points of epileptic childrens' behavioral problems, sleep-related behavioral disorders, breathe problems, frequency rate of wake up at nights, sweating during sleep, long lasting fall a sleep time, difficulty on fall asleep, nightmare problems, feeling weary after sleep, somnambulism scores were higher than the ones of their healthy siblings. Also as the epileptic group were evaulated according to their antiepileptic treatment we did not find a significant difference between the different antiepileptic treatments.

Conclusions Sleep disturbances frequently faces in the epileptic facts and there are few studies about this issue. As indicated in our study, sleep structure is not normal in epileptic patients eventhough seizures are under control by treatment. The evaluation of sleep disturbances should be the main part of further treatment of epileptic patients.

\section{SLEEPING BRUXISM AND OROFACIAL DYSFUNCTION IN CHILDREN AND ADOLESCENTS}

doi:10.1136/archdischild-2012-302724.1819

'MBD Gavião, 'ABM Montes, '2PM Castelo, 'F Kobayashi, 'MCS Marquezin. 'Department of Paediatric Dentistry, University of Campinas - UNICAMP, Piracicaba; ${ }^{2}$ Department of Biological Sciences, Federal University of São Paulo, UNIFESP, São Paulo, Brazil

Background and Aim Sleep bruxism (SB) is a parafunctional oromotor habit that can pose a threat to the integrity of the masticatory system. Thus, this study aimed to evaluate temporomandibular disorders (TMD) and orofacial dysfunction in children and adolescents with $\mathrm{SB}$, taking on a case-control study design.

Methods Three hundred and sixteen subjects aged from 7 to 17 years were examined. From these, 52 presented SB (Case Group) according to parent's report about teeth grinding or clenching at least three time a week and presence of dental wear facets according clinical examination. The Control Group was composed by 104 gender/ age/dentition phase-matched subjects. The mean age was $10.86 \pm 2.32$ years. TMD was evaluated according to the Research Diagnostic Criteria for Temporomandibular Disorders (RDC/TMD) Axis I. For orofacial dysfunction diagnosis, it was used The Nordic Orofacial Test Screnning (NOT-s). The data were analyzed using descriptive statistics, Shapiro-Wilks, unpaired $t$ test, Mahn-Whitney, Qui-square and Fisher's exact tests, when indicated, considering $\alpha=0.05$.

Results The prevalence of SB was higher in boys (60\%) than girls $(P<0.05)$. The presence of TMD was similar in both groups $(P>0.05)$. Subjects with SB presented NOT-S scores significant higher than subjects without $\mathrm{SB}(P=0.002)$, as well as girls in relation to boys in Case $(P=0.010)$ and Control $(P=0.019)$ groups for NOT-s interview. Habits, impairment in chewing, in swallowing and in masticatory muscles were determinant factors upon orofacial dysfunction.

Conclusions TMD was not implied in BS, but the orofacial dysfunction could be considered influencing factor in children and adolescents with SB.

\section{FACTORS ASSOCIATED WITH OBJECTIVE SLEEP PATTERN IN SCHOOL AGE CHILDREN LIVING IN TEHRAN CITY}

doi:10.1136/archdischild-2012-302724.1820

${ }^{1,2} \mathrm{~A}$ Saber Gharamaleki, ${ }^{3} \mathrm{~A}$ Zamani, ${ }^{4} \mathrm{~K}$ Jaafarian. ${ }^{1}$ Faculty of Health and Nutrition, Students' Research Committee, Tabriz University of Medical Science, Tabriz; ${ }^{2}$ Department of Nutrition and Biochemistry, School of Public Health: ${ }^{3}$ Tehran University of Medical Sciences; ${ }^{4}$ Department of Nutrition and Biochemistry, School of Public Health, Tehran University of Medical Sciences, Tehran, Iran

Objective Our objective was to identify factors associated with sleep pattern in school age children living in Tehran city.

Study Design This was a cross-sectional study of 6-9 year olds children ( $\mathrm{n}=270)$ who recruited by multistage sampling among primary school in Tehran city. Sleep pattern and efficiency was measured for 7 consecutive days using Actigraph accelerometer. Height and weight were directly measured using standardized equipment. Other factors including age, gender, history of preterm birth, birth weight, playing video game, watching TV, family income and parental educational level gathered by standard questionnaire.

Results 7days and weekdays sleep duration was significantly shorter $(p<0.05)$ in older children while there was not the same for weekend sleep duration. After adjusting for age we found no significant associations between measured factors and sleep pattern. There was no significant gender difference for sleep pattern, but girls had better $(p<0.05)$ sleep efficiency during 7 days and weekdays compared to boys.

Conclusions Sleep efficiency showed an inverse correlation with sleep duration. No significant relationship was found between sleep efficiency during 7 days, weekdays and weekend with the personal and the environmental factors.

\section{SLEEP REPORTED COMPLAINTS WITH PARENTS AMONG THE PEDIATRIC BETWEEN 3 MONTHS AND 14 YEARS OLD PRESENTED IN IRANIAN PEDIATRICS}

doi:10.1136/archdischild-2012-302724.182

S Afsharpaiman. Health Research Center, Baqiyatallah University of Medical Sciences, Tehran, Iran

Background Every disruption in the start, continue or decrease of quantity or quality of the sleep are considered as sleep disorders. The major cause of sleep disorders in children are temporary and spontaneously improve.

Methods In a cross-sectional study, children who presented to the Najmiyeh hospital in Tehran during 2009 and 2010 were enrolled Children 3 months to 14 years with any chronic disorders who were referred for routine screening and vaccination in this age range were included and the data was collected by using a questionnaire included demographic and clinical. The data were analyzed using SPSS software version 17 th by statistical frequency, chi-square, Student $t$ test and Mantle Hanzel tests and P.value $<0.05$ was considered significant.

Results The 301 children were studied. Mean age was $26.60 \pm 31.59$ months with a standard deviation. The study population sex ratio, male to female equal to $53.3 \%$ and $46.4 \%$ is equivalent to 161 to 140 $55.48 \%$ of the parents brought the child with complaints of sleep The underling variables including sex, age, affect the prevalence of parent report sleep disorders (P.value $<0.05$ )

Conclusion We suggest that further programmed evaluations are needed to evaluate children according to their age and the children should be assessed for sleep disorders in any range of the ages.

1822 EFFECT OF NEEDLE LENGTH FOR RESPONSE TO HEPATITIS B VACCINE IN MACROSOMICNEONATES: A PROSPECTIVE RANDOMIZED STUDY

doi:10.1136/archdischild-2012-302724.1822 
'R Ozdemir, 'FE Canpolat, 'S Yurttutan, 'MY Oncel, '0 Erdeve, 1,2U Dilmen. 'Zekai Tahir Burak Maternity Teaching Hospital; ${ }^{2}$ Pediatrics, Yildirim Beyazit University, Faculty of Medicine, Ankara, Turkey

Background and Aims The objective of this study was to determine whether use of a longer (1 in.) rather than astandard (5/8 in.) needle used for macrosomic neonates (birthweight over $4000 \mathrm{~g}$ ) may affect antibodytiters after immunization against hepatitis $B$ virus (HBV).

Methods Fifty nine healthy infants were vaccinated at birth, 1 and 6 months of age with hepatitis Bvaccine, with follow up to 7 months of age. Infants were randomized into two groups according to needlelength of first vaccine at birth. First group vaccinated with standart needle length and other group receivedvaccine by longer needle length.

Results Macrosomic infants who were immunized with a longer needle achieved significantly higher antibodytiters to hepatitis B surface antigen than standart needle length (median, 3890.2 vs $1311.7 \mathrm{mIU} / \mathrm{mL}$, respectively; $\mathrm{p}=0.001$ ).

Conclusions Macrosomic neonates benefit from longer needle length with higher levels of antibody titersafter $\mathrm{HBV}$ vaccination.

\section{THE EVALUATION OF ANTIBODY RESPONSES IN STEROID SENSITIVE CHILDREN WITH NEPHROTIC SYNDROME}

doi:10.1136/archdischild-2012-302724.1823

${ }^{1} \mathrm{O}$ Can, ${ }^{1} \mathrm{Fi}$ Arkan, 'MA Taşar, ${ }^{2 B}$ C Celikel Acar, ${ }^{3} \mathrm{E}$ Can, ${ }^{1} Y$ Dallar Bilge. 'Pediatrics; ${ }^{2}$ Pediatric Nephrology, Ministry of Health, Ankara Training and Education Hospital; ${ }^{3}$ Biochemistry, Ministry of Health, Etlik Obstetrics-Gynecology Training and Education Hospital, Ankara, Turkey

Nephrotic syndrome is characterized by massive proteinüria, hypoalbuminemia, and edema. Immunoglobulin changes, $T$ lymphocyte function disorders, and the reduction in complement levels in the nephrotic syndrome cause an increase in the risk of viral and bacterial infections.

For this purpose, children with nephrotic syndrome followed in the Pediatric Nephrology Department were screened for antibody levels and seroconversion of hepatitis A, hepatitis B, chicken pox, mumps, measles, rubella, and pneumococcus vaccines.

An evaluation of the seroconversion status of study and control groups revealed that all the children had negative anti CMV IgM, anti HCV, anti HIV, anti HAV IgM, HBsAg, antimumps IgM, antimeasles IgM and antirubella IgM. Only two children in the study group had anti-VZV IgM positive.

When the study and control groups were evaluated within the groups separately, a statistically significant difference was observed in the positivity of anti HAV IgG, antiHBs, anti pneumococ IgG, anti VZV IgG, antimumps IgG, antimeasles IgG and antirubella IgG before and after vaccination.

When the study and control groups were compared to each other in respect to antibody titers before and after vaccination, there was no significant difference in anti HAV IgG, anti HBs, antipneumococcus IgG, antimumps IgG, antimeasles IgG and antirubella IgG. But the study groups were statically different in respect to anti VZV IgG.

The evaluation of children with nephrotic syndrome for the seroconversion status and their vaccination against the necessary microorganisms would be a cost effective approach reducing the frequency of relapses and infection related morbidity and mortality.

\section{THE EVALUATION OF THE VACCINATION RATE AND THE PARENTS KNOWLEDGE ABOUT VACCINATION}

doi:10.1136/archdischild-2012-302724.1824

M Inalhan, A Ozlem, T Ozlem, F Feyza, C Mehmet, P Esra. Zeynep Kamil Maternity and Childrens' Disease Training and Research State Hospital, Istanbul, Turkey
Objective Immunisation is one of the most important weapons for protecting individuals and the community from serious diseases.

Method The survey method is applied to the mothers of children 12 months and over who hospitalized in our clinic between Februray and May 2010. Mothers of children who agreed to participate in the study and whose vaccine records can be reached are included in the study.

Findings The average age of the children was $4.56 \pm 2.5$ years, of the mothers was $29.23 \pm 4.74$ and of the fathers was $32.93 \pm 5.47$. The most common answer given to the question "Why vaccinate?" was "for being healty" ( $\mathrm{n}=35)$. The most memorable vaccine was tuberculous vaccine (\%55). In our study, we didn't find any statistically significant difference between the immunization status of children and the mother's education, mother's profession, father's profession, occupational distribution.

There wasn't any difference between the vaccination status of children and the age average of mothers: $(z=-0,430 ; p=0,668)$ the age average of fathers $(z=0,756 ; p=0,450)$.

Results Despite all studies and campaigns, the rate of being full vaccinated is beneath the levels of \%90-95. We need more campaigns to increase the vaccination rate of the society.

\section{INACTIVATED-TRIVALENT INFLUENZA VACCINATION IN ASTHMATIC UNDER-5 CHILDREN: A RANDOMIZED DOUBLE-BLIND PLACEBO TRIAL}

doi:10.1136/archdischild-2012-302724.1825

'F Fayyaz Jahani, 'M Karramyyar, 'E Ahmadnezhad. 'Urmia University of Medical Sciences, Urmia; ${ }^{2}$ Tehran University of Medical Sciences, Tehran, Iran

Background There are very little evidences that influenza vaccination reduced asthma exacerbation in under-5 children and the risk of vaccination is still being discussed.

This study aimed to detect the effect of influenza vaccination on symptomatic in asthmatic under- 5 .

Methods A balanced RCT with 140 asthmatic day-care children with stable situation (6 to 60 months yrs), which were vaccinated with either one-dose Inactivated-trivalent Influenza vaccine or placebo was performed. They participated for only one influenza season and were followed every two weeks. We recorded when symptom scores reached a predefined severity level.

Results Exacerbation rate among vaccinated and un-vaccinated were $13 \%$ and $53 \%$, respectively $(R R=0.24,95 \% C I=0.01-0.34) .48 .6 \%$ of vaccinated and $768 \%$ of placebo group reported cough $(\mathrm{RR}=0.61$, $95 \% \mathrm{CI}=0.04-0.35)$. The rate of wheezing report were $20 \%$ in vaccinated and $68.6 \%$ in unvaccinated group $(\mathrm{RR}=0.25,95 \% \mathrm{CI}=0.02-2.01)$ The RR for dispenea was 0.36 with $95 \% \mathrm{CI}$ that equal 0.1 to 3.65 .

Conclusion In this trial we demonstrated that tolerability and efficacy of the trivalent inactivated product in under- 5 children. Then this results support annual influenza vaccination in children with asthma

\section{SURVIVAL RATE OF DISSEMINATED BCGITIS IN CHILDREN WITH PRIMARY IMIMUNODEFICIENCY - SINGLE CENTER EXPERIENCE}

doi:10.1136/archdischild-2012-302724.1826

'D Al-Zahrani, ${ }^{2} \mathrm{~A}$ Shehzah, ${ }^{2} \mathrm{~S}$ Al Tubaiti, ${ }^{3} \mathrm{M}$ Bakhash, ${ }^{3} \mathrm{M}$ Azzam, ${ }^{3} \mathrm{~A}$ Abu Taleb, ${ }^{4} \mathrm{~S}$ Bin Yaheb, ${ }^{3} \mathrm{~A}$ Abdulwahab, ${ }^{3} \mathrm{~J}$ Yousef, ${ }^{3} \mathrm{~S}$ Abdulmalik, ${ }^{3} \mathrm{~N}$ Hussein, ${ }^{3} \mathrm{~A}$ Moben Alhaq,

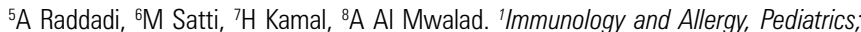
${ }^{2}$ Pediatrics; ${ }^{3}$ Pediatric ICU; ${ }^{4}$ Pediatric Surgery; ${ }^{5}$ Dermatology Department; ${ }^{6}$ Pathology Department; 'Pediatric Infectious Disease; ${ }^{8}$ Microbiology/Immunology-Laboratory Medicine, King Abdulaziz Medical City-WR, Jeddah, Saudi Arabia

Background and Aims Overall prevalence of primary immunodeficiency (PID) is 1:2,000 live births. PID characterized by increased 